\title{
Focal delivery of AAV2/1-transgenes into the rat brain by localized ultrasound-induced BBB Opening
}

\section{Angelika Alonso1, Eileen Reinz ${ }^{2}$, Barbara Leuchs², Jürgen Kleinschmidt'2, Marc Fatar'1, Bart Geers ${ }^{3}$, Ine Lentacker ${ }^{3}$, Michael G. Hennerici ${ }^{1}$, Stefaan C. de Smedt ${ }^{3}$ and Stephen Meairs ${ }^{1}$}

${ }^{1}$ Department of Neurology, Universitätsmedizin Mannheim, University of Heidelberg, Mannheim, Germany; ${ }^{2}$ German Cancer Research Center, Heidelberg, Germany; ${ }^{3}$ Laboratory of General Biochemistry \& Physical Pharmacy, Ghent University, Gent, Belgium

\author{
Ankur Tyagi \\ Department of Anesthesia and Intensive Care, PGIMER, Chandigarh
}

\section{Background}

Blood brain barrier (BBB) is one of the major components of central nervous system (CNS) which prevents all kinds of external molecules to infest CNS. ${ }^{1}$ However this protection also prevents gene and targeted drug delivery to CNS. The tight junctions of BBB pose as an impermeable wall for components to penetrate within the BBB. ${ }^{2}$ Recently an upcoming approach of utilizing Ultrasound with ultrasound contrast components is of intrest to researchers as it allows the penetration of these components without damaging neuronal cell. Using the above technique till now antibodies or plasmid DNA has been successfully delivered into rodents. ${ }^{3}$

\section{Study Design}

The vector plasmid encoding for AAV2 terminal repeat elements and the transgene nIsLAcZ (nuclear localization signal; $\beta$-galactosidase) driven by a CMV promoter was created. For opening of BBB $500 \mathrm{KHZ}$ transducer was used. It was positioned stereotacticaly over the wistar rat and one hemisphere was isonated. Transducer was run by wave form generator and amplifier. To validate BBB opening the rat had to undergo MRI scan of brain. Gulodinum a chemical which do not pass the intact BBB in histochemical sections was found to be located in transfected cells of CNS. Immunohistochemistery and RTPCR quantification were done to analyse the transfection of vector plasmid into the rat brain.

\section{Implications}

Authors describe the targeted delivery of AAV into BBB by ultrasound. It opens up new paths and possibilities to treat disorders linked to CNS. However further studies on animal models are required to validate the technique having immense potential in clinical therapy.

doi : 10.5214/ans.0972.7531.210107

Reference

1. Meairs $\mathrm{S}$ and Alonso $\mathrm{A}$. Ultrasound, microbubbles and the blood-brain barrier. Prog Biophys Mol Biol 2007; 93: 354-362.

2. Pardridge, WM. The blood-brain barrier: bottleneck in brain drug development. NeuroRx 2005; 2: 3-14.

3. Alonso A, Reinz E, Fatar M, et al. Neurons but not glial cells overexpress ubiquitin in the rat brain following focused ultrasound-induced opening of the blood-brain barrier. Neuroscience 2010; 169: 116-124. 